\title{
Parabolic class number of a subgroup of the normalizer of $\Gamma_{0}(m)$
}

\author{
Refik Keskin
}




\title{
PARABOLIC CLASS NUMBER OF A SUBGROUP OF THE NORMALIZER OF $\Gamma_{0}(m)$
}

\author{
REFIKK KESKINN
}

Received 4 May, 2011

\begin{abstract}
In this study, we introduce a subgroup of the normalizer of $\Gamma_{0}(\mathrm{~m})$ and we calculate parabolic class number of this group.
\end{abstract}

2000 Mathematics Subject Classification: 20H10; 20H20

Keywords: normalizer, parabolic class number, Fuchsian group, orbit

\section{INTRODUCTION}

Let $m$ be a positive integer and let $N\left(\Gamma_{0}(m)\right)$ be the normalizer of $\Gamma_{0}(m)$ in $\operatorname{PSL}(2, \mathbb{R})$. The normalizer $N\left(\Gamma_{0}(m)\right)$ was studied for the first time by Newman in [8]. A complete description of the elements of $N\left(\Gamma_{0}(m)\right)$ is given in [2]. Especially, a necessary and sufficient condition for $N\left(\Gamma_{0}(m)\right)$ to act transitively on $\hat{\mathbb{Q}}$ $=\mathbb{Q} \cup\{\infty\}$ was given in [1]. Moreover, the signatures and therefore the parabolic class numbers of some subgroups of $N\left(\Gamma_{0}(m)\right)$ were calculated in [1], [5], and [6] . In this study, we introduce a subgroup of $N\left(\Gamma_{0}(m)\right)$ and we calculate the parabolic class number of this subgroup when $m$ is a square free positive integer.

\section{PRELIMINARies}

Let $\Lambda$ be a discrete subgroup of $\operatorname{PSL}(2, \mathbb{R})$. When $x \in \mathbb{R} \cup\{\infty\}$ is a fixed point of a parabolic element of $\Lambda$, we say that $x$ is a parabolic point of $\Lambda$. We also call a parabolic point of $\Lambda$, a cusp of $\Lambda$.

The proof the following theorem is easy and can be found in [7] or [10].

Theorem 1. Let $\Lambda$ be a discrete subgroup of PSL $(2, \mathbb{R})$ and assume that $\Lambda^{*}$ is a subgroup of finite index in $\Lambda$. Let $P$ be the set of the parabolic points of $\Lambda$. Then $P$ is the set of the parabolic points of $\Lambda^{*}$ and the parabolic class number of $\Lambda^{*}$ is the number of orbits of $\Lambda^{*}$ on $P$. 
By a Fuchsian group $\Lambda$ we will mean a finitely generated discrete subgroup of $\operatorname{PSL}(2, \mathbb{R})$ the group of conformal homeomorphisms of the upper-half plane. The most general presentation for $\Lambda$ is

Generators:

$$
\begin{gathered}
a_{1}, b_{1}, \cdots, a_{g}, b_{g} \text { Hyperbolic } \\
x_{1}, x_{2}, \cdots, x_{r} \text { Elliptic } \\
p_{1}, p_{2}, \cdots, p_{s} \text { Parabolic }
\end{gathered}
$$

with the relations

$$
x_{1}^{m_{1}}=x_{2}^{m_{2}}=\ldots . .=x_{r}^{m_{r}}=\prod_{i=1}^{g}\left[a_{i}, b_{i}\right] \prod_{j=1}^{r} x_{j} \prod_{k=1}^{s} p_{k}=1 .
$$

We then say $\Lambda$ has signature ( see [4] )

$$
\left(g, m_{1}, m_{2}, \ldots \ldots, m_{r} ; s\right) \text {. }
$$

$s$ is the number of parabolic classes, i.e., of parabolic generators, and $r$ is the number of elliptic generators. The $m_{i}$ are the periods of $\Lambda$.

A complete description of the elements of $N\left(\Gamma_{0}(m)\right)$ is given in [2]. If we represent the elements of $N\left(\Gamma_{0}(m)\right)$ by the associated matrices, then the normalizer consists exactly of the matrices

$$
\left(\begin{array}{cc}
a e & b / h \\
c m / h & d e
\end{array}\right)
$$

where $e \mid\left(m / h^{2}\right)$ such that $\left(e, m / h^{2} e\right)=1$ and $h$ is the largest divisor of 24 for which $h^{2} \mid m$ with the understanding that the determinant of the matrix is $e>0$. If $e \mid n$ and $(e, n / e)=1$, we represent this as $e \| n$ and we say that $e$ is exact divisor of $n$. Thus we have

$$
N\left(\Gamma_{0}(m)\right)=\left\{\left(\begin{array}{cc}
a \sqrt{q} & b / h \sqrt{q} \\
c m / h \sqrt{q} & d \sqrt{q}
\end{array}\right): \operatorname{det}=1 ; 1 \leq q ; q \| \frac{m}{h^{2}} ; a, b, c, d \in \mathbb{Z}\right\} .
$$

The following theorem is given in [1] and [11].

Theorem 2. Let $m$ have prime power decomposition $2^{\alpha_{1}} 3^{\alpha_{2}} p_{3}^{\alpha_{3}} \ldots . p_{r}^{\alpha_{r}}$. Then $N\left(\Gamma_{0}(m)\right)$ acts transitively on $\hat{\mathbb{Q}}$ if and only if $\alpha_{1} \leq 7, \alpha_{2} \leq 3, \alpha_{i} \leq 1, i=3,4, \ldots, r$.

If $m$ is a square free positive integer, then

$$
N\left(\Gamma_{0}(m)\right)=\left\{\left(\begin{array}{cc}
a \sqrt{q} & b / \sqrt{q} \\
c m / \sqrt{q} & d \sqrt{q}
\end{array}\right): \operatorname{det}=1 ; 1 \leq q ; q \| m ; a, b, c, d \in \mathbb{Z}\right\} .
$$


Let $\Gamma$ be modular group. In [3], we calculated parabolic class number of the subgroup $\Gamma_{0}(n) \cap \Gamma^{N}$ such that

$$
\Gamma^{N}=\left\{\left(\begin{array}{cc}
a & b \\
c & d
\end{array}\right) \in \Gamma: a \equiv d \equiv 0(\bmod N) \operatorname{or} b \equiv c \equiv 0(\bmod N)\right\}
$$

and

$$
\Gamma_{0}(n)=\left\{\left(\begin{array}{ll}
a & b \\
c & d
\end{array}\right) \in \Gamma: c \equiv 0(\bmod n)\right\},
$$

where $(N, n)=1$ and $N$ is a power of a prime number. And it is shown that parabolic class number of this group is

$$
N \sum_{d \mid n} \varphi\left(\left(d, \frac{n}{d}\right)\right)
$$

Let $(m, n)=(m, N)=(n, N)=1$ and let $\Gamma_{0}^{*}(n)$ be defined by

$$
\Gamma_{0}^{*}(n)=\left\{\left(\begin{array}{cc}
a \sqrt{q} & b / h \sqrt{q} \\
c m / h \sqrt{q} & d \sqrt{q}
\end{array}\right) \in N\left(\Gamma_{0}(m)\right): c \equiv 0(\bmod n)\right\}
$$

and let $\Gamma(m, N)$ be defined by

$$
\Gamma(m, N)=\left\{A \in N\left(\Gamma_{0}(m)\right): a \equiv d \equiv 0(\bmod N) \text { or } b \equiv c \equiv 0(\bmod N)\right\} .
$$

Moreover, we define $\Gamma_{0}^{*}(m, n, N)$ by $\Gamma_{0}^{*}(m, n, N)=\Gamma(m, N) \cap \Gamma_{0}^{*}(n)$. It is clear that $\Gamma_{0}^{*}(m, n, 1)=\Gamma_{0}^{*}(n)$. In this paper, we will show that the parabolic class number of $\Gamma_{0}^{*}(m, n, N)$ is

$$
N \sum_{d \mid n} \varphi\left(\left(d, \frac{n}{d}\right)\right)
$$

when $(m, n)=(m, N)=(n, N)=1$ and $N$ is a power of a prime number. In the next section, we give five lemmas, which are necessary for our main theorem.

\section{MAIN THEOREMS}

From now on, otherwise stated, we will assume that $m$ is a square free positive integer and $(m, n)=(m, N)=(n, N)=1$.

Lemma 1. $\left|N\left(\Gamma_{0}(m)\right): \Gamma_{0}^{*}(m, n, N)\right|=\frac{\left|\Gamma_{0}(m): \Gamma_{0}(m n N)\right|}{2}$

Proof. Let $q \mid m$. Since $m$ is square free, $(q, m / q)=1$. Thus it follows that $\left(q N^{2},(m / q) n\right)=1$. Therefore there exist two integers $x_{1}$ and $y_{1}$ such that $q N^{2} x_{1}-(m n / q) y_{1}=1$. Let

$$
A_{q}=\left(\begin{array}{cc}
N \sqrt{q} & y_{1} / \sqrt{q} \\
m n / \sqrt{q} & N x_{1} \sqrt{q}
\end{array}\right) .
$$

Then $A A_{q}^{-1} \in \Gamma_{0}(m n N)$ for any 


$$
A=\left(\begin{array}{cc}
a N \sqrt{q} & b / \sqrt{q} \\
c m n / \sqrt{q} & d N \sqrt{q}
\end{array}\right) \in \Gamma_{0}^{*}(m, n, N) .
$$

Thus $A \in \Gamma_{0}(m n N) A_{q}$. On the other hand, since $\left(q, n(m / q) N^{2}\right)=1$, there exist two integers $x_{2}$ and $y_{2}$ such that $q x_{2}-n(m / q) N^{2} y_{2}=1$. Let

$$
B_{q}=\left(\begin{array}{cc}
x_{2} \sqrt{q} & y_{2} N / \sqrt{q} \\
m n N / \sqrt{q} & \sqrt{q}
\end{array}\right) .
$$

Then we see that $B B_{q}^{-1} \in \Gamma_{0}(m n N)$ for any

$$
B=\left(\begin{array}{cc}
a \sqrt{q} & b N / \sqrt{q} \\
c m n N / \sqrt{q} & d \sqrt{q}
\end{array}\right) \in \Gamma_{0}^{*}(m, n, N) .
$$

Thus it follows that $B \in \Gamma_{0}(m n N) B_{q}$. It can be seen that $\Gamma_{0}(m n N) A_{q_{1}}$ $\neq \Gamma_{0}(m n N) A_{q_{2}}$ and $\Gamma_{0}(m n N) B_{q_{1}} \neq \Gamma_{0}(m n N) B_{q_{2}}$ for $q_{1} \neq q_{2}$. Moreover,

$$
\Gamma_{0}(m n N) A_{q_{1}} \neq \Gamma_{0}(m n N) B_{q_{2}}
$$

for $q_{1} \mid m$ and $q_{2} \mid m$. Thus it follows that $\left|\Gamma_{0}^{*}(m, n, N): \Gamma_{0}(m n N)\right|<\infty$ and it can be shown that $\left|\Gamma_{0}^{*}(m, n, N): \Gamma_{0}(m n N)\right|=2^{t+1}$ where $t$ is the number of the prime divisor of $m$. Also we have

$$
\begin{aligned}
\mid N\left(\Gamma_{0}(m)\right) & : \Gamma_{0}^{*}(m, n, N) \mid=\frac{\left|N\left(\Gamma_{0}(m)\right): \Gamma_{0}(m n N)\right|}{\left|\Gamma_{0}^{*}(m, n, N): \Gamma_{0}(m n N)\right|} \\
& =\frac{\left|N\left(\Gamma_{0}(m)\right): \Gamma_{0}(m)\right|\left|\Gamma_{0}(m): \Gamma_{0}(m n N)\right|}{2^{t+1}}
\end{aligned}
$$

Since $\left|N\left(\Gamma_{0}(m)\right): \Gamma_{0}(m)\right|=2^{t}$, the proof follows.

In view of the above lemma, it is seen that $\Gamma_{0}^{*}(m, n, N)$ is finitely generated and therefore it is a Fuchsian group.

Lemma 2. Let $N=p^{r}$ where $p$ is a prime number and let $k / s \in \hat{\mathbb{Q}}$ with $p \mid s$. Then there exist some $T \in \Gamma_{0}^{*}(m, n, N)$ such that $T(k / s)=k_{1} / s_{1}$ with $\left(s_{1}, N\right)=1$.

Proof. Since $(m, N)=(n, N)=1$, we see that $\left(m n, N^{2}\right)=1$ and therefore there exist two integers $x$ and $y$ such that $N^{2} x-m n y=1$. Let

$$
T=\left(\begin{array}{cc}
x N & y \\
m n & N
\end{array}\right) .
$$

Then $T \in \Gamma_{0}^{*}(m, n, N)$ and $T(k / s)=\frac{x N k+y s}{m n k+s N}=k_{1} / s_{1}$ with $s_{1}=m n k+s N$. It can be seen easily that $\left(s_{1}, N\right)=1$.

Lemma 3. Let $(k, s)=(s, N)=1$. Then there exist some $T \in \Gamma_{0}^{*}(m, n, N)$ such that $T(k / s)=k_{1} / s_{1}$ with $s_{1} \mid n$. 
Proof. Let $s_{1}=(s, n)$ and $q=(s, m)$. Then $s_{1}=(s, k n)=(s, k n N)$. Moreover $(s, m / q)=1$, since $m$ is square free. Therefore $\left(s, k n N \frac{m}{q}\right)=s_{1}$ and thus there exist two integers $c_{1}$ and $d_{1}$ such that $s d_{1}+k n \frac{m}{q} N c_{1}=s_{1}$. Since $\left(d_{1},\left(k n m / s_{1} q\right) N\right)=1$, there exist some integer $k_{0}$ such that

$$
\left(d_{1}-\frac{k n m}{s_{1} q} N k_{0},(m / q) n N^{2}\right)=1 .
$$

Let

and

$$
d=d_{1}-\frac{k n m}{s_{1} q} N k_{0}
$$

$$
c=c_{1}+\frac{s}{s_{1}} k_{0} .
$$

Then $k n(m / q) N c+s d=s_{1}$. On the other hand, $\left(d q, c N^{2}(m / q) n\right)=1$ since $(d, c)=$ $\left(d,(m / q) n N^{2}\right)=1$. It can be seen easily that $(q, c)=1$. Therefore $\left(d q, c(m / q) n N^{2}\right)$ $=1$. Thus there exist two integers $x$ and $y$ such that $x d q-c(m / q) n N^{2} y=1$. Let

$$
T=\left(\begin{array}{cc}
x \sqrt{q} & y N / \sqrt{q} \\
c m n N / \sqrt{q} & d \sqrt{q}
\end{array}\right) .
$$

Then $T \in \Gamma_{0}^{*}(m, n, N)$ and

$$
T(k / s)=\frac{q x k+s N y}{c k n m N+d s q}=\frac{q x k+s N y}{q(c k n(m / q) N+d s)}=\frac{x k+N(s / q) y}{(c k n(m / q) N+d s)}=\frac{k_{1}}{s_{1}} .
$$

Lemma 4. Let $\left(a_{1}, d_{1}\right)=\left(a_{2}, d_{1}\right)=1$ and $d_{1} \mid n$. Then $a_{1} / d_{1}$ is conjugate to $a_{2} / d_{1}$ under $\Gamma_{0}^{*}(m, n, N)$ if and only if $a_{1} \equiv a_{2}(\bmod N t)$ where $t=\left(d_{1}, n / d_{1}\right)$.

Proof. Suppose that $a_{1} / d_{1}$ is conjugate to $a_{2} / d_{1}$ under $\Gamma_{0}^{*}(m, n, N)$. Then there exist some $T \in \Gamma_{0}^{*}(m, n, N)$ such that $T\left(a_{1} / d_{1}\right)=a_{2} / d_{1}$. Assume that

$$
T=\left(\begin{array}{cc}
a \sqrt{q} & b N / \sqrt{q} \\
c m n N / \sqrt{q} & d \sqrt{q}
\end{array}\right),
$$

where $q \mid m$. Then we have

$$
\frac{a a_{1} q+b N d_{1}}{c n m N a_{1}+d d_{1} q}=\frac{a_{2}}{d_{1}}
$$

and

$$
a d q-b c n(m / q) N^{2}=1 .
$$

Since

$$
\begin{gathered}
a\left(c m n N a_{1}+d d_{1} q\right)-\frac{c m n N}{q}\left(a a_{1} q+b d_{1} N\right)=d_{1}, \\
d q\left(a a_{1} q+b d_{1} N\right)-b N\left(c m n N a_{1}+d d_{1} q\right)=a_{1} q,
\end{gathered}
$$


and $\left(d_{1}, a_{1} q\right)=1$, we see that $\left(a a_{1} q+b d_{1} N, c m n N a_{1}+d d_{1} q\right)=1$. Therefore there exist some $u= \pm 1$ such that

$$
u a_{2}=a a_{1} q+b N d_{1}
$$

and

$$
u d_{1}=c m n N a_{1}+d d_{1} q .
$$

Since $q\left(c n(m / q) N a_{1}+d d_{1}\right)=u d_{1}$ and $\left(q, d_{1}\right)=1$, it follows that $q=1$. Thus

$$
\begin{gathered}
u a_{2}=a a_{1}+b N d_{1}, \\
u d_{1}=c m n N a_{1}+d d_{1},
\end{gathered}
$$

and

$$
a d-b c n m N^{2}=1
$$

From the above equations, we see that $u a_{2} \equiv a a_{1}(\bmod t N), u \equiv d(\bmod t N)$, and $a d \equiv 1(\bmod t N)$. Therefore $a_{1} \equiv u^{2} a_{2}(\bmod t N)$, which implies that $a_{1} \equiv a_{2}(\bmod$ $t N)$. Now assume that

$$
T=\left(\begin{array}{cc}
a N \sqrt{q} & b / \sqrt{q} \\
c m n / \sqrt{q} & d N \sqrt{q}
\end{array}\right),
$$

where $a d N^{2} q-b c n(m / q)=1$. Then we obtain

$$
\frac{a a_{1} N q+b d_{1}}{c n m a_{1}+d d_{1} q N}=\frac{a_{2}}{d_{1}} \text {. }
$$

Similarly, it is seen that $\left(c n m a_{1}+d d_{1} q N, a a_{1} N q+b d_{1}\right)=1$. Thus there exist some $u= \pm 1$ such that

$$
u a_{2}=a a_{1} N q+b d_{1}
$$

and

$$
u d_{1}=c m n a_{1}+d d_{1} q N .
$$

Since $u d_{1}=q\left(c n(m / q) a_{1}+d d_{1} N\right)$ and $\left(q, d_{1}\right)=1$, it follows that $q=1$. Thus

$$
\begin{gathered}
u a_{2}=a a_{1} N+b d_{1}, \\
u d_{1}=c m n a_{1}+d d_{1} N,
\end{gathered}
$$

and

$$
a d N^{2}-b c n m=1 \text {. }
$$

From the above equations, we get

$$
\begin{gathered}
u a_{2} \equiv b d_{1}(\bmod N), \\
u d_{1} \equiv \operatorname{cmna}_{1}(\bmod N),
\end{gathered}
$$

and

$$
b c m n \equiv 1(\bmod N) .
$$

This implies that $a_{1} \equiv a_{2}(\bmod N)$. In the same way we see that

$$
u a_{2} \equiv a a_{1} N(\bmod t),
$$




$$
u \equiv d N(\bmod t)
$$

and

$$
a d N^{2} \equiv 1(\bmod t)
$$

Thus we have $a_{1} \equiv a_{2}(\bmod t)$. Since $(t, N)=1$, it follows that $a_{1} \equiv a_{2}(\bmod t N)$.

Now suppose that $a_{1} \equiv a_{2}(\bmod t N)$, where $t=\left(d_{1}, n / d_{1}\right)$. Let $n_{1}=n / d_{1}$. Then $t=\left(d_{1}, n_{1}\right)$ and $\left(a_{1} a_{2}, d_{1}\right)=1$. Therefore $t=\left(a_{1} a_{2} n_{1}, d_{1}\right)$. This shows that $t N=$ $\left(d_{1} N, a_{1} a_{2} n_{1} N\right)$. Since $t N \mid a_{2}-a_{1}$, there exist two integers $x$ and $y$ such that $n_{1} a_{1} a_{2} N x+d_{1} N y=a_{2}-a_{1}$. Thus we obtain $a_{1}\left(n_{1} a_{2} N x+1\right)+d_{1} N y=a_{2}$. If we take $b=y$ and $a=n_{1} a_{2} N x+1$, we have $a a_{1}+d_{1} N b=a_{2}$. Let $c=n_{1} d_{1} x=n x$ and $d=1-N n_{1} a_{1} x$. Then $c N a_{1}+d d_{1}=d_{1}$. On the other hand, we see that

$$
\begin{aligned}
a d-b c N^{2} & =a\left(1-N n_{1} a_{1} x\right)-b n_{1} d_{1} x N^{2} \\
& =a-\left(a a_{1}+d_{1} N b\right) N n_{1} x=a-n_{1} a_{2} N x=1 .
\end{aligned}
$$

Let

$$
T(z)=\frac{a z+b N}{c m N z+d}
$$

Then $n \mid c$ and therefore $T \in \Gamma_{0}^{*}(m, n, N)$. Moreover, we get

$$
T\left(a_{1} / d_{1}\right)=\frac{a a_{1}+b d_{1} N}{c N a_{1}+d d_{1}}=a_{2} / d_{1} .
$$

The proof of the following lemma is easy and will be omitted.

Lemma 5. Let $\left(a_{1}, d_{1}\right)=\left(a_{2}, d_{1}\right)=1, d_{1}\left|n, d_{2}\right| n$, where $d_{1}$ and $d_{2}$ are positive integers. If $a_{1} / d_{1}$ is conjugate to $a_{2} / d_{2}$ under $\Gamma_{0}^{*}(m, n, N)$, then $d_{1}=d_{2}$.

In the following, we give a lemma without proof, which we use later. The lemma appears in [9], page 73, as problem.

Lemma 6. Let $m$ and $k$ be positive integers. Then the number of the positive integers $\leq m k$ that are prime to $m$ is $k \varphi(m)$.

Theorem 3. Let $p$ be a prime number and $N=p^{r}$. Then the parabolic class number of $\Gamma_{0}^{*}(m, n, N)$ is

$$
N \sum_{d \mid n} \varphi\left(\left(d, \frac{n}{d}\right)\right)
$$

Proof. It suffices to calculate the number of orbits of $\Gamma_{0}^{*}(m, n, N)$ on $\hat{\mathbb{Q}}$. By Lemma 4 and Lemma 6, there exist $N \varphi((d, n / d))$ different orbits for each $d \mid n$. Then from Lemma 2 to Lemma 5, it follows that the number of orbits of $\Gamma_{0}^{*}(m, n, N)$ on $\hat{\mathbb{Q}}$ is

$$
\sum_{d \mid n} N \varphi\left(\left(d, \frac{n}{d}\right)\right)=N \sum_{d \mid n} \varphi\left(\left(d, \frac{n}{d}\right)\right)
$$


Corollary 1. Let $p$ be a prime number. Then the parabolic class number of $\Gamma\left(m, p^{k}\right)$ is $p^{k}$.

Corollary 2. The parabolic class number of $\Gamma_{0}^{*}(n)$ is

$$
\sum_{d \mid n} \varphi\left(\left(d, \frac{n}{d}\right)\right)
$$

Let $r$ be a natural number and let

$$
\Gamma_{0}^{+}(r)=\left\{\left(\begin{array}{cc}
a \sqrt{q} & b / \sqrt{q} \\
c r / \sqrt{q} & d \sqrt{q}
\end{array}\right): a d q-b c r / q=1 ; 1 \leq q ; q \| r ; a, b, c, d \in \mathbb{Z}\right\} .
$$

Then $\Gamma_{0}^{+}(r)$ is a subgroup of the normalizer of $\Gamma_{0}(r)$. Moreover, any element of $\Gamma_{0}^{+}(r)$ is an Atkin-Lehner involution of $\Gamma_{0}(r)$. Recall that an Atkin-Lehner involution $w_{q}$ of $\Gamma_{0}(r)$ is an element of determinant 1 of the form

$$
w_{q}=\left(\begin{array}{cc}
a \sqrt{q} & b / \sqrt{q} \\
c r / \sqrt{q} & d \sqrt{q}
\end{array}\right)
$$

for some exact divisor $q$ of $r$. If $h=1$, then $\Gamma_{0}^{+}(r)$ is equal to $N\left(\Gamma_{0}(r)\right)$, where $h$ is the largest divisor of 24 such that $h^{2} \mid r$. The following lemmas are well known and their proofs can be found in [11].

Lemma 7. $\Gamma_{0}^{+}(r)$ acts transitively on the set $\hat{\mathbb{Q}}=\mathbb{Q} \cup\{\infty\}$ if and only if $r$ is a square-free positive integer.

Lemma 8. Let $r=m / h^{2}$. Then $N\left(\Gamma_{0}(m)\right)$ acts transitively on the set $\hat{\mathbb{Q}}$ if and only if $\Gamma_{0}^{+}(r)$ acts transitively on the set $\hat{\mathbb{Q}}$.

Let $r=m / h^{2}$. Then, it is well-known and easy to see that

$$
N\left(\Gamma_{0}(m)\right)=\left(\begin{array}{cc}
1 / \sqrt{h} & 0 \\
0 & \sqrt{h}
\end{array}\right) \Gamma_{0}^{+}(r)\left(\begin{array}{cc}
1 / \sqrt{h} & 0 \\
0 & \sqrt{h}
\end{array}\right)^{-1} .
$$

Assume that $N\left(\Gamma_{0}(m)\right)$ acts transitively on the set $\hat{\mathbb{Q}}$. Taking $r=m / h^{2}$ and

$$
X=\left(\begin{array}{cc}
1 / \sqrt{h} & 0 \\
0 & \sqrt{h}
\end{array}\right),
$$

it is immediate that $X H X^{-1}$ is equal to $\Gamma_{0}^{*}(m, n, N)$, provided that $H=\Gamma_{0}^{*}(r, m, N)$. Therefore, we can give the following corollary easily.

Corollary 3. Suppose that $N\left(\Gamma_{0}(m)\right)$ acts transitively on the set $\hat{\mathbb{Q}}$ and $N=p^{k}$ for some prime $p$. Then parabolic class number of $\Gamma_{0}^{*}(m, n, N)$ is

$$
N \sum_{d \mid n} \varphi\left(\left(d, \frac{n}{d}\right)\right)
$$




\section{REFERENCES}

[1] M. Akbas and D. Singerman, "The signature of the normalizer $\Gamma_{0}(N)$," in Groups, combinatorics and geometry. Proceedings of the L.M.S. Durham symposium, held July 5-15, 1990 in Durham, UK, ser. Lond. Math. Soc. Lect. Note Ser., M. Liebeck, Ed., vol. 165. Cambridge: Cambridge University Press, 1992, pp. 77-86.

[2] J. H. Conway and S. P. Norton, "Monstrous moonshine," Bull. Lond. Math. Soc., vol. 11, pp. 308-339, 1979.

[3] R. Keskin, “A note on some modular subgroups," Rocky Mt. J. Math., vol. 35, no. 2, pp. 573-585, 2005.

[4] R. Keskin and B. Demirtürk, "On suborbital graphs for the normalizer of $\Gamma_{0}(N)$," Electron. $J$. Comb., vol. 16, no. 1, p. 18, 2009.

[5] M.-L. Lang, “The signature of $\Gamma_{0}^{+}(n)$, , J. Algebra, vol. 241, no. 1, pp. 146-185, 2001.

[6] C. Maclachlan, "Groups of units of zero ternary quadratic forms," Proc. R. Soc. Edinb., Sect. A, vol. 88, pp. 141-157, 1981.

[7] T. Miyake, Modular forms. Transl. from the Japanese by Joshitaku Maeda. Berlin: SpringerVerlag, 1989.

[8] M. Newman, "Conjugacy, genus, and class numbers," Math. Ann., vol. 196, pp. 198-217, 1972.

[9] I. Niven, H. S. Zuckerman, and H. L. Montgomery, An introduction to the theory of numbers. 5th ed. New York: John Wiley \& Sons, Inc., 1991.

[10] G. Shimura, Introduction to the arithmetic theory of automorphic functions, ser. Publications of the Mathematical Society of Japan. Princeton, NJ: Publishers and Princeton University Press, 1971, vol. 11.

[11] D. Singerman, "Subgroups of Fuchsian groups and finite permutation groups," Bull. Lond. Math. Soc., vol. 2, pp. 319-323, 1970.

\section{Author's address}

\section{Refik Keskin}

Sakarya University, Mathematics Department, TR 54187 Sakarya, Turkey

E-mail address: rkeskin@sakarya.edu.tr 